\title{
Low Complexity Business Status Update Framework: One Touch Approach for Food and Beverage Industry
}

\author{
E. M. Ang \\ Faculty of Information Science \& Technology, Multimedia University, Malacca, 75450, Malaysia \\ Email: jessicaaem@live.com \\ K. S. Yong \\ Faculty of Information Science \& Technology, Multimedia University, Malacca, 75450, Malaysia \\ Email: kinsum91@gmail.com \\ Lenard Z. W. Lee \\ Faculty of Information Science \& Technology, Multimedia University, Malacca, 75450, Malaysia \\ Email: lenardlee90@gmail.com \\ K. K. Wee \\ Faculty of Information Science \& Technology, Multimedia University, Malacca, 75450, Malaysia \\ Email: wee.kuok.kwee@mmu.edu.my
}

\begin{abstract}
Today, many individuals are used to dine-out. However, they are unaware of the business operation on that particular moment of the day. Several times, we end up arriving at the restaurant only to find that it is closed/having a break. Hence, we propose a framework for a system of related applications which solves the above problem by being informative regarding the business operability to the customers. Firstly, a trader side framework that allows food stall operators to inform the status and nature of their business to their customers whether they are open for business or not. Secondly, a customer side framework for the food stall operator customers to view restaurant status, menu and to place booking. Mobile applications are developed based on the proposed framework for both trader and customer. And lastly, a website is developed for the general public to view the business status of the stall operators. By being able to inform customers the status of the business, it will provide convenience to many people in our society. Our contribution will be the aforementioned framework as well as mobile apps and website which provides convenience to many people in our society, in terms of reducing time wastage as well as fuel costs to the stall's destination.
\end{abstract}

Index Terms-Framework, android application, website, business operation, sensor tag.

\section{INTRODUCTION}

These days' people tend to dine-out by having a meal with their family, colleagues, or individually, as it saves time and energy from the meal preparation. It is considered to be convenient to people with a hectic lifestyle, especially those that are living in the city, where people do not have the time, tired or reluctant to set up a proper meal after a long day's work. This is especially so during festive season.

Based on an online newspaper article in [1], it is stated that based on the Malaysian Food Barometer (MFB) survey with a sample of 2000 people, the percentage of Malaysians dine outside of home for at least a meal is 64 percent while the other 36 percent that have their meals at home, 12.5 percent have at the minimum one meal outside. Figure 1 gives an overview of the Malaysian food cultures eating habits [2]. Based on a recent study as stated in [1], Professor Doctor Jean-Pierre Poulain, whom is a head researcher, Taylor's Toulouse University Centre co-director and leader of Chair of Food Studies, stated that many people in Malaysia dines out. He added that this occurs in places like Kuala Lumpur as well as the countryside.

As a customer or even tourist that wants to dine out, often we would try to figure out what to eat every meal. As of today, we often utilize mobile devices frequently on daily affairs due to efficiency as well as trend [3]. In the android market, there are existing mobile applications which gives a bunch of choices and selections on various cuisines [4]. There are also mobile applications which provide delivery services. For example, fast food services like McDelivery [5]. Apart from mobile applications, customers are able to browse online for information on restaurants that are well established. However, some smaller stalls which have yummy food, for example hawker stalls, are not made known themselves. These 
stalls rely on customers to advertise their business and bring about more customers. Also, their business operation location and time is not fixed as they tend to be mobile and flexible. For example, a 'char kuey teow' (fried noodles) stall that goes around various 'pasar malam' (night market) to do business. Thus, we proposed a framework which involves several applications that is able to bridge this gap between the food traders and customers. The applications include android mobile applications, one for the trader, and one for the client, as well as a website for both users to have access on any mobile device.

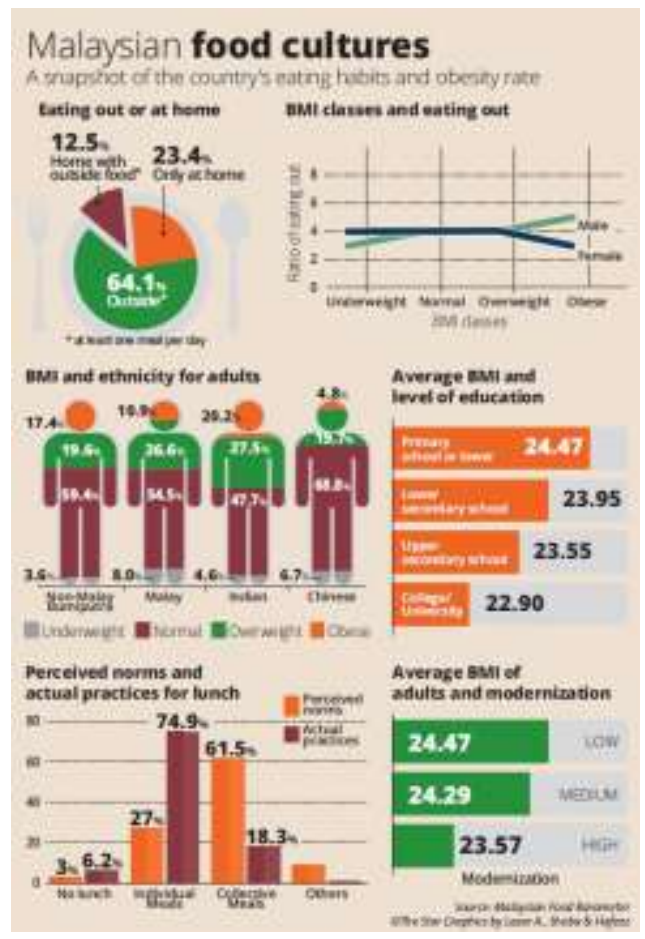

Fig.1. Malaysian Food Culture [2].

The flow of this manuscript is arranged as the following: Section II gives a brief elaboration on the related works made available in the Google Play market. The problem statement and objective of this development is given in Section III and Section IV respectively. Section $\mathrm{V}$ provides an overview of the applications architecture's framework. The Bluetooth sensor setup for traders is elaborated in Section VI, while Section VII provides an overview of the client side framework. Section VIII gives a brief description on the development of the websites outlook. Section XI explains in detail in regards to the server application. In the last section, we conclude the paper.

\section{RELATED WORK}

Several related works which are made available on Google Play are reviewed [4]. An android app called Galaxy Eat Out was presented by a Thailand company, Wongnai Media Company Limited, which allows users to have a choice over 100'000 restaurants to dine in Bangkok and a few provinces in Thailand [6]. This app also provides direction and contact information of the restaurant. In addition, it is an interactive application as it allows users to post images and reviews. Figure 2 illustrates the icon of Galaxy Eat Out. Apart from android app, Wongnai also has their very own webpage that provides several features which include a choice of various places to dine, restaurants information, coupons and etc. [7].

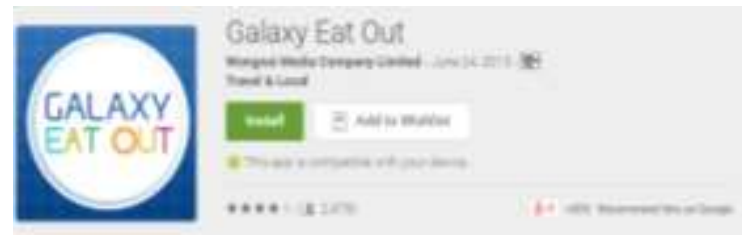

Fig.2. Galaxy Eat Out [5].

Another android application that is made known both in Singapore and Malaysia is the app called HungryGoWhere [8][9]. Figure 3 and 4 respectively shows what the android application looks like for Singapore and Malaysia on Google Play.

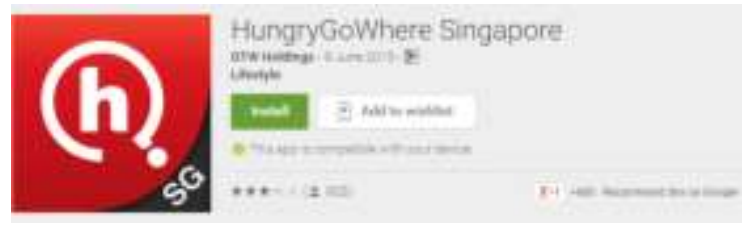

Fig.3. HungryGoWhere Singapore [8].

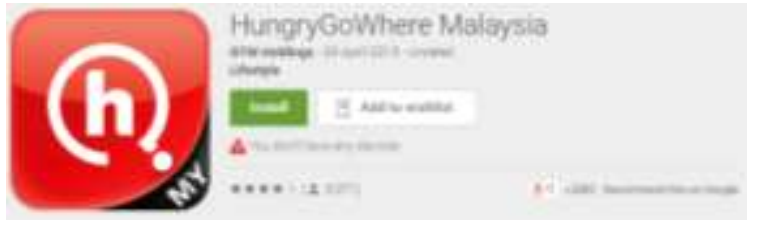

Fig.4. HungryGoWhere Malaysia [9].

HungryGoWhere is developed not only android applications but also iOS applications. They have a website for viewers to browse as well [10]. This android application acts as a food guide assisting users to determine what and where to eat around them, as well as what to cook. Apart from that, this app has a filter function which allows users to filter the list of places to dine based on price, popularity (in terms of dish or restaurant), cuisine, and halal [9]. Through the app, users can connect and share with other users their findings. In addition, the Singapore version of this app provides the feature for users to place table bookings [8].

In [11] displays an android application called Nearby Restaurants Café Pizza. Figure 5 is an image of the app on Google Play.

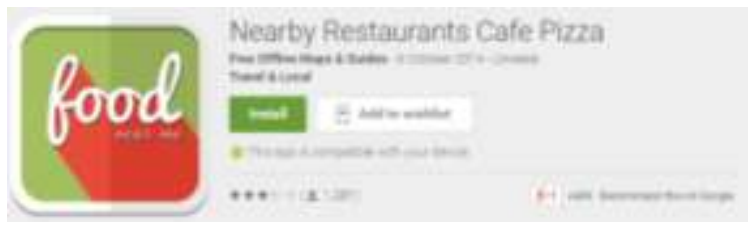

Fig.5. Nearby Restaurants Café Pizza [11]. 
This app determines nearby fast food services. It calculates the distance between the users mobile through GPS to the nearest fast food restaurant. However, the range covered by this app does not include Malaysia. Apart from fast food services, several other types of places are supported. This includes restaurants, bars, café, food (shops), night clubs, parking areas, automated teller machine (ATM), and bakeries. Information about the location is provided in terms of the name of the restaurant, the distance to get there, navigation, contact number, as well as ratings.

Another android application in [12] features to provide users with local details about food, shopping, relaxation as well as promotional deals. This app is based on location, hence users are able to look for items near them with the map provided [12]. Similarly to the Hungrygowhere app, a website is developed for users to view on browsers. This app provides one stop destination in determining business and listings, posting free ads, and etc.

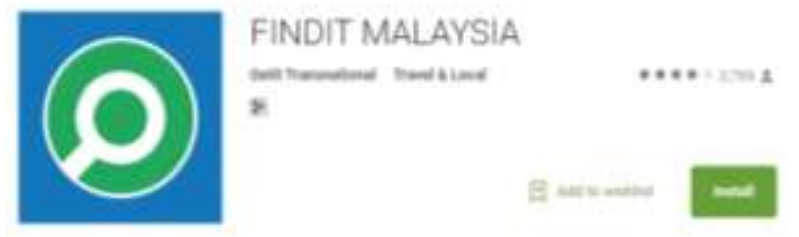

Fig.6. FINDIT Malaysia [12].

Recently, the popular social media, Facebook, has added a function to their 'page info' [13][14]. Businesses, like cafes, restaurants or shops, can now display their stalls operation hours. Based on the set operation days, a status is displayed 'Closed now' on non-operating hours or days, and 'Open' during the operating time. Figure 7 illustrates on the app made available on Google Play for users to install.
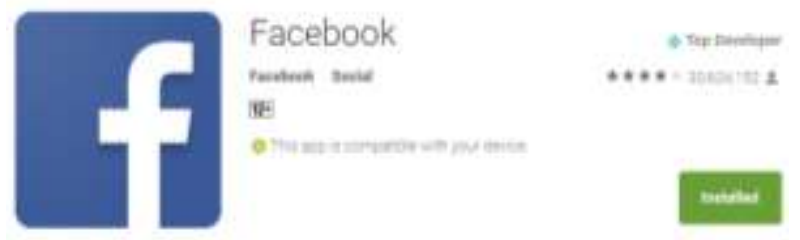

Fig.7. Facebook [13].

\section{PROBlem Statement}

A common occurrence for people who eat most of their meals out is discovering that the food stall is close or not ready for business upon reaching it. This consumes a lot of time and petrol which can be avoided if people knew the opening status of the stall before beginning their journey there. Sometimes, due to unavoidable circumstances, the food stall may not be open. The operator of these stalls would also like to have a good way of informing the public that they are closed. This can also reduce the disappointment as well as stress level from spending several hours on travelling, including getting caught in a traffic jam, just to end up finding that the stall is closed.

Based on current app development, the focus are more towards well established restaurants and businesses. However, small food stalls which sell yummy food are not made known. Hence, there is a need to bridge the gap between the rural (whom are mostly not technology savvy) and urban community.

\section{OBJECTIVES}

To propose a framework and develop an application which enables the general public to be informed about the opening status of food operators and also make orders/reservations via Internet.

To propose a framework and develop an application for food stall operators to update their stall's status (opening, closing and etc), and receive order/reservations.

\section{FRAMEWORK OF THE APPLICATIONS ARCHITECTURE}

Figure 8 is an overview of the projects architecture. This diagram is to visualize the system's framework and understand how the food stall operators (trader) and the food stall operators customers (client) are able to interact with the developed android application via network connection. Both mobile applications are android based mobile applications which are developed using the Android Software Development Kit (Android SDK) and installed as an .apk on trader and client devices. Android SDK is utilized as it consists of development tools, an emulator, source codes and several libraries which are made available for building Android applications [15].

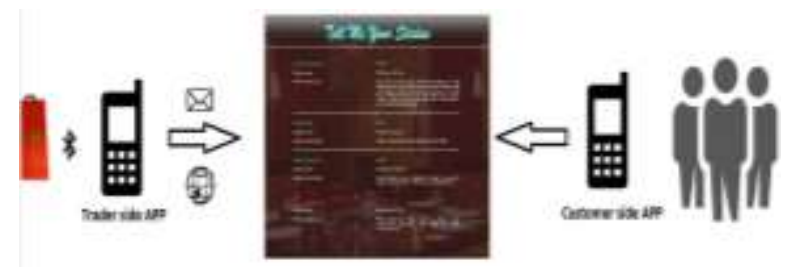

Fig.8. Architecture Overview.

The website was designed primarily on Hypertext Preprocessor (PHP) and functions to display the operating status of the food stall operators to the public and for the public to place their orders.

Figure 9 represents the flow chart of the applications architecture for both trader and client. Both users are required to register before being able to login. As for the traders, the registration comes together with the Bluetooth setup. Once logged in, both users are able to view the Stall List, Menu, and Stall Details/Information page however, trader has additional functions which include Insert Food Items, Delete Food Items and view Booking List. As for the client, only the clients are able to perform Orders/Reservations. Further elaboration on the frameworks setup and functionality are given in the sections below. 


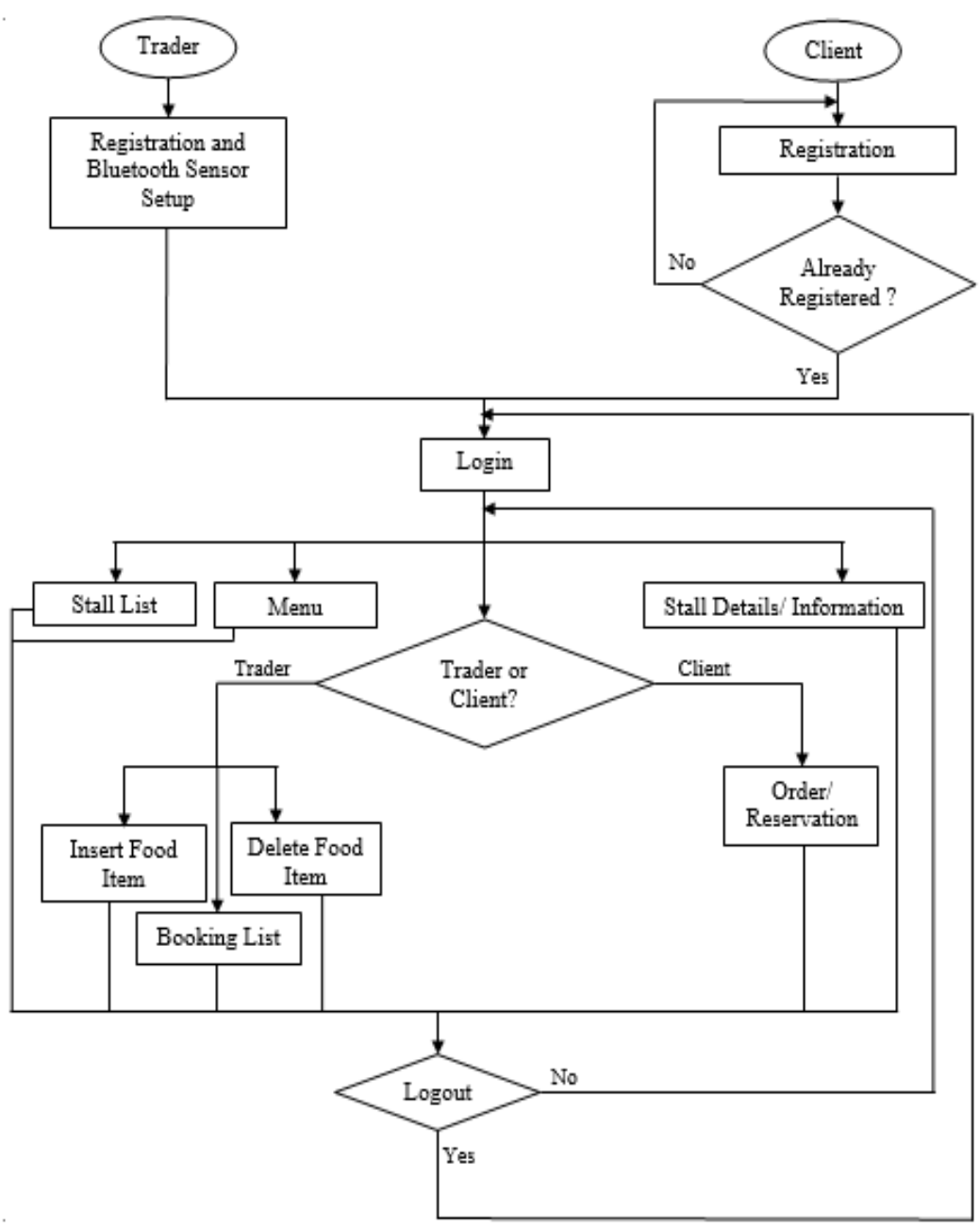

Fig.9. Flow Chart.

\section{Bluetooth Sensor Setup (TRADER Side)}

In order connect the trader side application to the Bluetooth sensor tag [16], the application needs to first

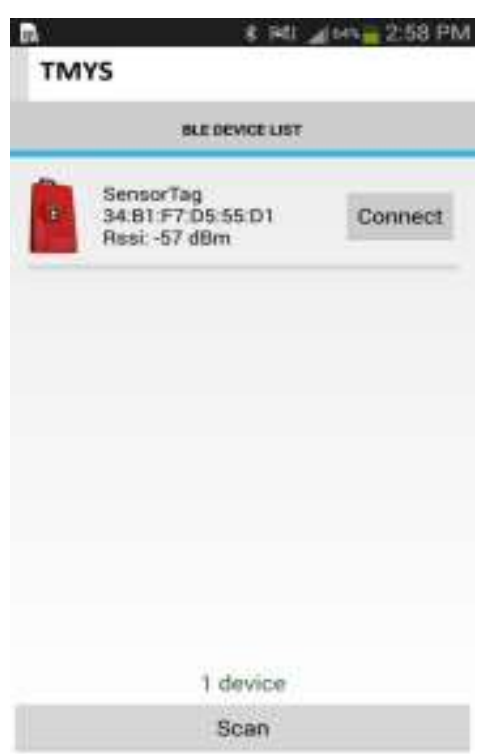

Fig.10. Bluetooth Sensor List scan for nearby sensor devices [17]. After scanning, if there are any devices in range, a list of devices will appear as in Figure 10 and the application can select which sensor to connect to.

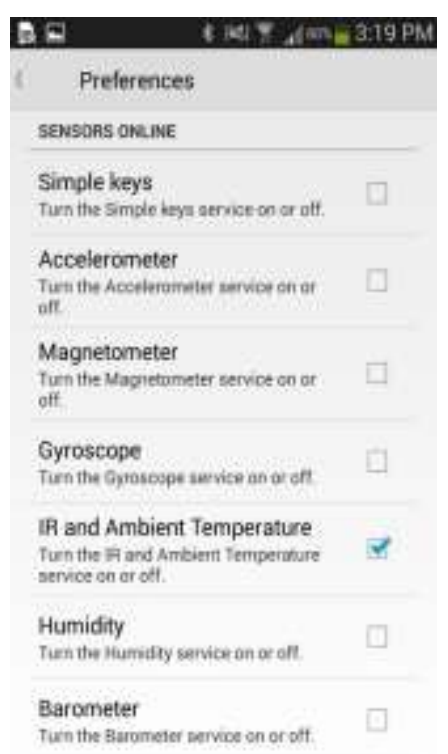

Fig.11. Sensor Parameters Selection 
The configuration and viewing of the environment variables, that is to be detected by the sensor, parameters can be selected from the preferences page. Users will need to press on the options button of their phone, and select the preference option and they will arrive at the page shown in Figure 11. Based on Figure 11, various environment variables can be selected to be detected for validating whether a stall is in operation or not.

Figure 12 shows the environment parameters and values detected by the sensor. As shown in Figure 6, the sensors are configured to detect object and ambience temperature. The values of the parameters constantly changes according to the values detected by the sensor. When the trader side application detects that the temperature is above a certain threshold due to cooking activity by the stall owner, it will send a Short Message Service (SMS) to the server to update the stall operating status from CLOSED to OPEN. After a certain period of inactivity, the temperature will revert to normal and the application will send another SMS to the server to update the status of the stall from OPEN to CLOSED.
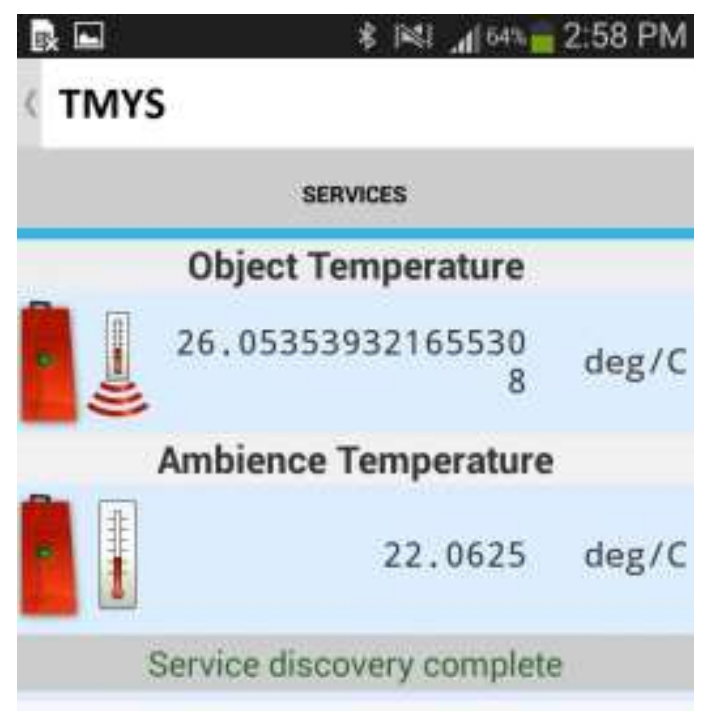

Fig.12. Sensor Parameters Detection

The android mobile application is developed using several Android APIs which can be looked-up in the package index [18]. Based on Figure 13, for the trader side mobile application, trader's stall is to be registered using the trader's mobile phone number simply by SMS. The SMS will be sent to our server via a GSM modem. Notification on stall registration will be sent out from our server to trader by SMS as well.

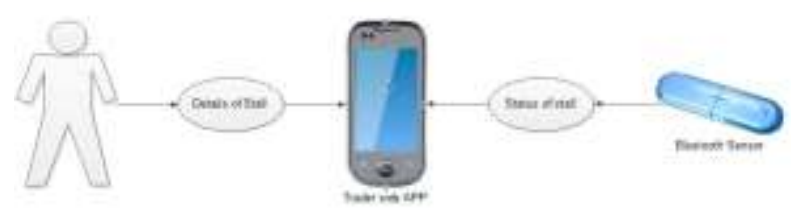

Fig.13. Trader Side Framework

The mobile app is connected to a Bluetooth sensor tag (Texas Instrument, n.d.-a; Texas Instrument, n.d.-b) whereby the sensor is configured to detect some elements in the environment which can indicate that a stall is open. As an example, the sensor can be configured to be sensitive to heat. The sensor can be placed close by kitchenware's that heats up when the stall is in operation. For example, kitchen stove and boiling pot heats up when the stall is in operation. The sensor can detect this significant increase of heat and automatically sends an update to the mobile device which in turn update the server to display the status of the stall as 'open'. When the cooking activity no longer happens, the temperature of the kitchen wares will revert back to normal. Upon detecting this, the sensor will again send an update to the mobile device and the mobile device can update the stall status on the web server to 'closed'. Apart from heat, the sensor can also be configured to detect motion, pressure and etc.

Traders have additional features whereby they can upload and remove images of their products to display as well as for reservation purposes. Traders will be able to view their client's details (mobile phone number, etc.) and orders on the booking list once the client has made an order.

\section{CLIENT SIDE FRAMEWORK}

Similarly to the trader side application, the client side application is also an android application system. Figure 14 gives an overview of the client side framework that's developed in a mobile android application for clients to view the stall's details (location, operation hours, etc.), status and menu of the trader's stall. By registering an account using their mobile phone number, clients are able to place an order/reservation in the android application. A login page is provided whereby clients can login to our application.

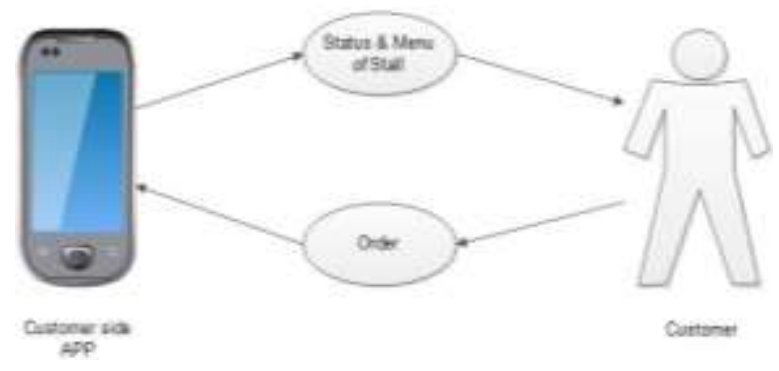

Fig.14. Client Side Framework

Using the client side mobile application, users can view the status of the stalls to know whether it is open or not. The client application retrieves this information from the server and displays it to the users. Clients are also able to perform booking of food via the application. At each restaurant page, clients are able to view the trader stall's food offers and select how many of food items that they would like to order. Traders are able to view the orders placed via the website. Once the booking has been made, for example in the case whereby the customer books a specific amount of food, the customer may proceed to the stall and inform the trader's their mobile 
number in order for the vendor to verify the booking. This cuts down on waiting time when the customer reaches the stall because the food stall owner is able to take their order much sooner.

\section{WEBSITE OUTLOOK}

In addition to the development, a website is developed using HyperText Markup Language (HTML), PHP and Cascading Style Sheets (CSS) [19]. Both trader and client website functions are similar to having use the android application, hence giving them an option to access the system through their web browsers on any device.

\section{SERVER APPLICATION}

In this project, server is utilized whereby it is connected to a Global System for Mobile (GSM) modem to receive SMS updates from stall operators. The wireless GSM modem needs to have a subscriber identity module (SIM) card, which can be easily obtained from a wireless carrier, in order to function in a GSM wireless network [20]. ActiveXperts Serial Port Component 3.2 [21] is utilized in the Attention Terminal (AT). It runs on commands which in this case, it is used to control the modulator-demodulator (MODEM) in order to have the functionality of sending and receiving SMS-es. This SMS update will tell if a particular stall is open or not. When a server receives a SMS from a trader side application, it will refer to the content of the SMS to update the status of the stall. If the content has the keyword "OPEN", then the server sets the stall status to open and updates the website accordingly. If the content has the keyword "CLOSED", then the server sets the stall status to be closed on the website.

\section{CONCLUSION}

In a nutshell, framework is developed to provide convenience to the society. The framework can be categorized as a Green Innovation as it is able to reduce time wastage, effort, and also fuel costs to the stall's destination. In big cities, traffic jams commonly occur during peak hours such as lunch time. When people have advance knowledge on the opening status of the eating location, they do not need to spend time travelling on the road just to discover that the place is not open after reaching their destination. The smaller food stalls with exquisite local delicacies can also be part of a choice for customers to try out, hence promoting our local cuisines worldwide.

Based on our proposed framework, stall operators have a good way of reaching out to the community to let the people know their operating status. This can also be a strategy to bridge between the high economic impact areas with the rural community and also the technological gap in the community. For example, low cost housing residents 'mak cik' (aunties) and 'pak cik' (uncles) whom are not tech savvy but cook delicious meals.

\section{REFERENCES}

[1] Tan, C. (2014). More Malaysians eat out nowadays but are we eating enough healthy food?. Retrieved from http://www.thestar.com.my/News/Nation/2014/08/03/Foo d-always-on-our-minds-More-Malaysians-eat-outnowadays-but-are-we-eating-enough-healthy-food/ on May 2015.

[2] Lazar, A., Shoba, \& Hafeez. (2014). Malaysian Food Cultures. Malaysian Food Barometer. Retrieved from http://www.thestar.com.my/ /media/Images/TSOL/Photos -Gallery/Nation/2014/08/03/malaysianfoodcultureschart 0308.ashx/ on May 2015.

[3] C. Zhou, Z. Weng, C. Xu, and Z. Su, "Integrated Traffic Information Service System for Public Travel Based on Smart Phones Applications: A Case in China," Int. J. Intell. Syst. Appl., vol. 5, no. 12, pp. 72-80, 2013.

[4] Google. (2012). Google Play. Retrieved from https://play.google.com/store/apps?hl=en on May 2015.

[5] McDonalds Malaysia. (2015). McDelivery Malaysia. Google Play. Retrieved from https://play.google.com/store/apps/details?id=my.com.mc donalds.delivery\&hl=en on May 2015.

[6] Wongnai Media Company Limited (2013). Galaxy Eat Out. Google Play. Retrieved from https://play.google.com/store/apps/details?id=com. wongn ai.samsung.eatout\&hl=en on July 2015.

[7] Wongnai Media Company Limited (2015). Wongnai. Google Play. Retrieved from https://www.wongnai.com/ on July 2015.

[8] GTW Holdings (2015). HungryGoWhere Singapore. Google Play. Retrived from https://play.google.com/store/apps/details?id=hgw.androi d.app on July 2015.

[9] GTW Holdings (2015). HungryGoWhere Malaysia. Google Play. Retrived from https://play.google.com/store/apps/details?id=com.singtel. hungrygowhere on July 2015.

[10] Singtel Digital Media Pte. Ltd. (2015). HungryGoWhere Malaysia. Retrieved from http://www.hungrygowhere.my/ on July 2015.

[11] Free Offline Maps \& Guides (2014). Nearby Restaurants Cafe Pizza. Google Play. Retrieved from https://play.google.com/store/apps/details?id=off.guide.on line.food on July 2015.

[12] Getit Transnational (2015). Findit Malaysia. Google Play. Retrieved from https://play.google.com/store/apps/details?id=com.kellton tech.maxisgetit on August 2015.

[13] Facebook (2015). Facebook. Google Play. Retrieved from https://play.google.com/store/apps/details?id=com.facebo ok.katana\&hl=en on August 2015.

[14] Facebook (2015). Facebook. Retrieved from http://www.facebook.com/ on August 2015.

[15] S.-C. Chong, B.-K. Leong, W.-K. New, and Y.-J. Chin, "An Android-based Remote Desktop for IOS Platforms," Int. J. Intell. Syst. Appl., vol. 5, no. 10, pp. 18-27, 2013.

[16] Texas Instrument. (n.d.-a). CC3541 SensorTag Development Kit. Retrieved from http://www.ti.com/tool/cc2541dk-sensor on May 2015.

[17] Texas Instrument. (n.d.-b). The SensorTag Story. Retrieved from

http://www.ti.com/ww/en/wireless_connectivity/sensortag 2015/index.html?DCMP=sensortag\&HQS=sensortag-bn on May 2015.

[18] Android Developers. (n.d.). Package Index|Android Developers. Retrieved from 
http://developer.android.com/reference/packages.html on May 2015.

[19] W3Schools. (n.d.). W3Schools Home. Retrieved from http://www.w3schools.com on May 2015.

[20] Developershome. (n.d.). Introduction to GSM / GPRS Wireless Modems. Retrieved from http://www.developershome.com/sms/GSMModemIntro.a sp on May 2015.

[21] ActiveXperts, S. (n.d.). ActiveXperts Serial Port Component. Retrieved from http://www.activexperts.com/serial-port-component/ on May 2015.

\section{Authors' Profiles}

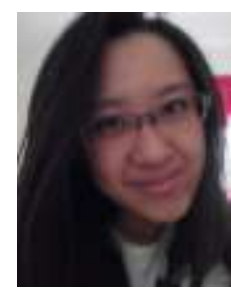

E. M. Ang was born in Malacca, Malaysia, in 1991. She obtained her Bachelor (Hons.) of Information Technology majoring in Security Technology from Multimedia University, Malacca, Malaysia, in the year 2013.

She is currently pursuing her Master degree in Science (Information Technology) in the very same university, performing research related to the Long Term Evolution (LTE) network. Her research interest includes areas in networking which involves the advancement of wireless communications technology.

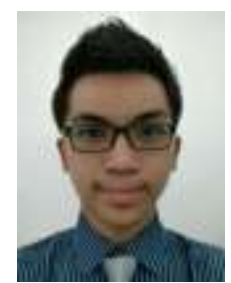

K. S. Yong received his Bachelor (Hons.) of Information Technology in Data Communications and Networking with First Class Honours from Multimedia University, Malacca, Malaysia in 2014.

$\mathrm{He}$ is currently a postgraduate student in Multimedia University, Malacca. His field of study is on Mobile Wireless Network, particularly in the area of power saving in the Long Term Evolution network.

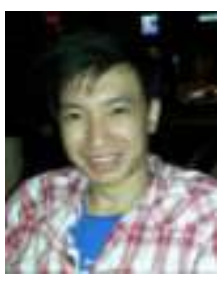

Lenard Z. W. Lee was born on August 11, 1990. He received his Bachelor (Hons.) of Information Technology from Multimedia University Malaysia in 2014.

$\mathrm{He}$ is currently pursuing his M. Sc (IT) at the same university. His research interest includes Long Term Evolution, WiMAX and machine learning.

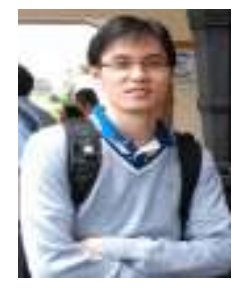

K. K. Wee received his BSc in Computer Science and MSc in Networking from University Putra, Kuala Lumpur, Malaysia. $\mathrm{He}$ then completed his study in $\mathrm{PhD}$ (Engineering) from Multimedia University, Malaysia.

$\mathrm{He}$ is currently Senior Lecturer at the Faculty of Information Science and Technology in Multimedia University, Melaka, Malaysia. He is also a member of Editor Board of an International journal and Senior Member of IEEE. His research interests include Quality of service, broadband wireless access, networking and mobile communication.
How to cite this paper: E. M. Ang, K. S. Yong, Lenard Z. W. Lee, K. K. Wee,"Low Complexity Business Status Update Framework: One Touch Approach for Food and Beverage Industry", International Journal of Intelligent Systems and Applications (IJISA), vol.7, no.12, pp.43-49, 2015. DOI: 10.5815/ijisa.2015.12.04 